\title{
Psicopatía versus trastorno antisocial de la personalidad
}

\author{
María José López Miguel \& María del Carmen Núñez Gaitán
} Universidad de Sevilla

Original Recibido el 23 de julio de 2008

\section{RESUMEN}

La psicopatía tal vez haga referencia a uno de los trastornos de la conducta más conocidos tanto a nivel clínico como entre la población general. De él se han dado múltiples definiciones a lo largo de su historia, sin embargo, se ha empleado, con cierta ligereza, para diagnosticar distintos tipos de comportamientos que en ocasiones tienen muy poco en común, pero que comparten los rasgos de frialdad y crueldad en su ejecución. De ahí que pueda afirmarse que, a pesar de su popularidad, en realidad es una gran desconocida en el ámbito de la Psicopatología. El objetivo de este trabajo, por lo tanto, es examinar el concepto de psicopatía y sus características más importantes. Asimismo, se trata de delimitar el concepto distinguiéndolo de otros afines y con los que a veces se ha confundido, como es el caso del trastorno antisocial de la personalidad.

Palabras clave: psicopatía, trastorno antisocial de la personalidad, sociopatía, trastorno de la personalidad, PCL-R. 
ABSTRACT

\section{Psychopathy versus antisocial personality disorder}

Psychopathy may be one of the best known behaviour disorders both at clinical level and in the general population but, given the many definitions it has had in its long history, it has often been used loosely to diagnose different types of behaviour that have very little in common except a display of coldness and cruelty. It may therefore be deduced that, despite its popularity, there are also many misconceptions surrounding psychopathology. The aim of this work is to examine the concept of psychopathy and its most important characteristics. In so doing, it attempts to clearly demarcate the concept of psychopathy from other similar conditions with which it is sometimes confused, such as antisocial personality disorder.

Key words: Psychopathy, antisocial personality disorder, sociopathy, personality disorders, $P C L-R$.

\section{Introducción}

El trastorno antisocial de la personalidad (TAP) es, tal vez, uno de los trastornos que más atracción ejerce sobre los profesionales de la salud mental y, en general, sobre el resto de la población, si bien su prevalencia es relativamente baja (entre $0^{\prime} 7-3 \%$, según la mayoría de los estudios).

El motivo de tal atracción podría ser el hecho de que los individuos que lo presentan muestran comportamientos que pueden ser catalogados de incomprensibles para la mayoría de los humanos. No cabe duda del carácter inexplicable que poseen actos como los que llevan a cabo algunos de estos sujetos, entre los que pueden mencionarse el canibalismo o el asesinato realizado de forma gratuita, sin motivaciones aparentes. O, incluso, acciones como el descuidar a sus hijos o robar (no tan infrecuente en nuestros días), pero con el fin de divertirse.

No obstante, a pesar de que es un trastorno del que todo el mundo ha oído hablar, que es de sobras conocido, no es menos cierto que su diagnóstico se efectúa con

Revista Española de Investigación Criminológica

Artículo 1, Número 7 (2009) $\quad$ www.criminología.net

ISSN: $1696-9219$ 
ligereza en ocasiones, pues cuando nos encontramos con alguien frío, distante, poco empático, con dificultades para mostrar su afecto, rápidamente se le coloca la etiqueta de psicópata; asimismo, cuando los medios de comunicación informan de un crimen ejecutado cruelmente y sin sentido, las sospechas recaen inmediatamente sobre alguien que, sin duda, debe mostrar una personalidad psicopática.

Todo esto implica que, a pesar de lo indicado, la psicopatía es una gran desconocida, por lo que se hace necesario aclarar a qué nos referimos cuando hablamos de este trastorno. Por ello, el objetivo del presente trabajo es realizar una revisión de las diversas definiciones que, desde el punto de vista de distintos autores, entre ellos algunos ya clásicos como Cleckley y Hare, se han propuesto.

\section{Definición de psicopatía}

En relación con los intentos de establecer una adecuada conceptualización del término psicopatía, puede afirmarse que no existe un acuerdo unánime ni respecto a la denominación, ni acerca del constructo o definición de psicopatía. Así, ha sido definido desde diversos puntos de vista, los cuales van desde el puramente biológico, hasta el más ambientalista.

Centrándonos en los autores "más contemporáneos", esto es, aquéllos que estudiaron el fenómeno de mediados del siglo XX en adelante, y dejando a un lado las definiciones propuestas a principios del siglo XIX, Karpman (1961) describió al psicópata como un individuo insensible, emocionalmente inmaduro, desdoblado y carente de profundidad, con emociones simples, sin capacidad para experimentar ansiedad o miedo, y sin la posibilidad de que las recompensas o castigos ejerzan efecto alguno sobre su comportamiento.

Sería interesante acudir a las descripciones que sobre la psicopatía realizaron autores como Cleckley (1941) y Hare (1984). Para el primero de estos autores, el síntoma básico de la psicopatía sería la deficiente respuesta afectiva hacia los demás, que explicaría el comportamiento antisocial que manifiesta el individuo. Además, son

Revista Española de Investigación Criminológica

Artículo 1, Número 7 (2009) $\quad$ www.criminología.net

ISSN: $1696-9219$ 
harto conocidos los criterios que propuso para definir al psicópata, la mayoría de los cuales siguen aceptándose en la actualidad, y que se muestran en la tabla 1.

Tabla 1. Criterios de Cleckley para describir la psicopatía (Cleckley, 1941)

1. Encanto externo y notable inteligencia

2. Inexistencia de alucinaciones y otras manifestaciones de pensamiento irracional

3. Ausencia de nerviosismo

4. Indigno de confianza

5. Mentiras e insinceridad

6. Falta de sentimientos de culpabilidad y de vergüenza

7. Conducta antisocial, sin aparente remordimiento

8. Razonamiento insuficiente y falta de capacidad para aprender de la experiencia vivida.
9. Egocentrismo patológico e incapacidad para amar

10. Gran pobreza de reacciones afectivas

11. Pérdida específica de intuición

12. Irresponsabilidad en las relaciones interpersonales

13. Comportamiento fantástico

14. Amenazas de suicidio raramente cumplidas

15. Vida sexual impersonal, trivial y poco integrada

16. Incapacidad para seguir cualquier plan de vida

Según este autor, al psicópata le falta la posibilidad de experimentar emociones, carece de ellas, aunque, en apariencia, se exprese de un modo normal. Es lo que denominó demencia o afasia semántica, que implica que los psicópatas son incapaces de entender y expresar el significado de experiencias emocionales, aún cuando puedan comprender el lenguaje (Millon y Davis, 2001).

Cleckley distinguía la psicopatía de la delincuencia ordinaria, a pesar de la existencia de indicadores de desinhibición comportamental en esta última (pobre juicio, comportamiento antisocial inmotivado), en función de indicadores de la afectividad (ausencia de vergüenza o sentimiento de culpa, egocentrismo e incapacidad para amar y pobreza general de afecto) e interpersonales (trato superficial, irresponsabilidad en las relaciones interpersonales y falsedad) (Hicks, Markon, Patrick, Krueger, y Newman, 2004). 
Hare (1991), por su parte, elabora una escala de estimación compuesta por 20 ítems, denominada Psychopathy Checklist Revised (PCL-R) en la que se incluyen dos factores que conforman la psicopatía, y que se exponen en la tabla 2.

Tabla 2. Factores que componen la psicopatía (Hare, 1991)

\begin{tabular}{|c|c|}
\hline Factor 1. Personalidad & Factor II. Desviación social \\
\hline $\begin{array}{l}\text { 1. Locuacidad/encanto superficial } \\
\text { 2. Grandioso sentimiento de autovalía } \\
\text { 4. Mentira patológica } \\
\text { 5. Manipulador } \\
\text { 6. Falta de remordimiento/culpa } \\
\text { 7. Afecto superficial } \\
\text { 8. Crueldad/falta de empatía } \\
\text { 16. No acepta responsabilidad de sus actos }\end{array}$ & $\begin{array}{l}\text { 3. Necesidad de estimulación } \\
\text { 9. Estilo de vida parásito } \\
\text { 10. Escaso autocontrol } \\
\text { 12. Precocidad en mala conducta } \\
\text { 13. Sin metas realistas } \\
\text { 14. Impulsividad } \\
\text { 15. Irresponsabilidad } \\
\text { 18. Delincuencia juvenil } \\
\text { 19. Revocación de la libertad condicional }\end{array}$ \\
\hline $\begin{array}{l}\text { Ítems adicionales que no pertenecen a los } \\
\text { factores }\end{array}$ & \\
\hline $\begin{array}{l}\text { 11. Conducta sexual promiscua } \\
\text { 17. Muchas relaciones maritales breves } \\
\text { 20. Versatilidad delictiva }\end{array}$ & \\
\hline
\end{tabular}

El primero de estos factores, denominado personalidad o desapego emocional (Patrick, 2000), incluye aquellos ítems que hacen referencia a características interpersonales y afectivas; el factor de desviación social, por su parte, abarca aquellos elementos relacionados con problemas de conducta, irresponsabilidad e impulsividad, y con un déficit en la socialización del individuo (Stalenhein, 2001); es decir, cada factor alude a cuestiones emocionales y comportamentales, respectivamente.

Revista Española de Investigación Criminológica

Artículo 1, Número 7 (2009) $\quad$ www.criminología.net

ISSN: $1696-9219$ 
Esta prueba ha demostrado ser un efectivo instrumento para distinguir individuos psicópatas de aquéllos que no lo son (Bolt, Hare, Vitale y Newman, 2004), pues incluye los dos factores ya mencionados (Garrido, 2005).

En general, y en opinión de Hare (1984), las definiciones y descripciones aportadas por numerosos autores tienen en común varios elementos. Por un lado, el elevado egocentrismo que caracteriza a los psicópatas, que encaja, asimismo, con su falta de empatía; por otro, su incapacidad para establecer relaciones afectivas con los demás.

Resumiendo, Garrido (2004) considera que un atributo esencial -considerado por muchos autores como rasgo común- es la disfunción afectiva en el ámbito interpersonal. Esta alteración de la afectividad se relaciona con rasgos tales como la insensibilidad, la falta de empatía y la ausencia de remordimientos, el egocentrismo, la mentira patológica y la manipulación. Esta alteración de la afectividad parece relacionarse con una débil activación del sistema defensivo, con un deseo de dominio y baja ansiedad. Entre las características comportamentales que definen al psicópata destaca este autor la reiterada violación de las normas sociales y la explotación de los demás $\mathrm{y}$, por último, judicialmente, se trata de una conducta delictiva persistente y particularmente violenta.

Sin embargo, recientes investigaciones plantean la posibilidad de que la psicopatía no se trate de una categoría discreta, sino de una dimensión continua, lo cual tendría importantes implicaciones para la evaluación, la investigación etiológica y el tratamiento de este trastorno (Marcus, John y Edens, 2004). A pesar de ello, no todos los psicópatas son iguales; las diferencias entre los subtipos de psicopatía podrían esperarse en términos de frecuencia, naturaleza e intensidad de los delitos cometidos: la ocurrencia de actos impulsivos y violentos puede esperarse con más frecuencia entre los psicópatas agresivos mientras que los actos de fraude y violencia instrumental son más característicos de los psicópatas estables (Hicks y cols., 2004).

Hare (1984) ha establecido una tipología del psicópata, diferenciando tres tipos: psicópata primario, secundario y disocial. El psicópata primario correspondería a la 
descripción realizada por Cleckley; el secundario o psicópata neurótico se caracterizaría por su capacidad para establecer relaciones afectivas, sentir culpa o remordimiento y por una elevada ansiedad; finalmente, el psicópata disocial sería un individuo de ambientes marginales con una subcultura propia, en el que también están presentes los sentimientos de culpa, lealtad y afecto, siendo su conducta antisocial debida a factores ambientales (Torrubia, 1987).

Igualmente, Eysenck (1981, 1995) distinguió entre psicopatía primaria y psicopatía secundaria. La psicopatía primaria, de la que es responsable la dimensión psicoticismo, se caracteriza por ausencia de sentimientos de culpabilidad, empatía o sensibilidad, y llevaría a los individuos cuyas puntuaciones en la misma sean altas a cometer actos delictivos con más probabilidad (Bueno, 1990), siendo éstos más agresivos (Chico y Ferrando, 1995; Eysenck y Eysenck, 1976). La asociación entre extraversión y neuroticismo daría lugar a la psicopatía secundaria (Pérez, 1986), en la que el individuo comete actos delictivos pero experimenta culpabilidad por ello.

Como puede observarse, estas tipologías ya estarían haciendo referencia a la existencia de diversas entidades, lo que ha llevado a la distinción entre psicopatía y trastorno antisocial de la personalidad.

Patrick (2000) y Blair (2003) establecen una diferenciación entre psicopatía y trastorno antisocial de la personalidad en los mismos términos que los autores anteriormente mencionados. Así, aunque sean considerados como un mismo trastorno, la psicopatía y el TAP no son semejantes, existiendo, por lo tanto, factores causales diferentes (si bien algunos de estos factores pueden ser comunes, como es el caso del aprendizaje). El primero de ellos equivaldría a la psicopatía primaria, ya descrita, caracterizada por déficit emocionales y afectivos, y por egoísmo; el segundo, por el contrario, podría ser comparable a la psicopatía secundaria, en la que se presentan alteraciones del comportamiento, concretamente en forma de actos antisociales, sin deterioro a nivel de las emociones. A continuación se profundizará más en este segundo tipo de psicopatía y en esta distinción entre ambos tipos. 


\section{Trastorno antisocial de la personalidad: concepto y características}

Siguiendo la definición del Manual Diagnóstico y Estadístico de los Trastornos Mentales en su cuarta edición revisada (APA, 2002), el Trastorno Antisocial de la Personalidad, también denominado psicopatía o sociopatía, se caracteriza por "un patrón general de desprecio y violación de los derechos de los demás, que comienza en la infancia o el principio de la adolescencia y continúa en la edad adulta” (p. 784). Los criterios para el diagnóstico del TAP pueden observarse en la tabla 3.

Tabla 3. Trastorno antisocial de la personalidad (APA, 2002)

CRITERIOS PARA EL DIAGNÓSTICO DEL TRASTORNO ANTISOCIAL DE LA PERSONALIDAD

A. Un patrón general de desprecio y violación de los derechos de los demás que se presenta desde la edad de 15 años, como lo indican tres (o más) de los siguientes ítems:

1. Fracaso para adaptarse a las normas sociales en lo que respecta al comportamiento ilegal, como lo indica el perpetrar repetidamente actos que son motivo de detención.

2. Deshonestidad, indicada por mentir repetidamente, utilizar un alias, estafar a otros para obtener un beneficio personal o por placer.

3. Impulsividad o incapacidad para planificar el futuro.

4. Irritabilidad y agresividad, indicados por peleas físicas repetidas o agresiones.

5. Despreocupación imprudente por su seguridad o la de los demás.

6. Irresponsabilidad persistente, indicada por la incapacidad de mantener un trabajo con constancia o de hacerse cargo de obligaciones económicas.

7. Falta de remordimientos, como lo indica la indiferencia o la justificación del haber dañado, maltratado o robado a otros.

B. El sujeto tiene al menos 18 años.

C. Existen pruebas de un trastorno disocial que comienza antes de la edad de 15 años.

D. El comportamiento antisocial no aparece exclusivamente en el transcurso de una esquizofrenia o un episodio maníaco. 
Para De Corral (1996) son los comportamientos impulsivos los que verdaderamente caracterizan este trastorno y que no permiten reparar en las consecuencias negativas de las conductas; también la ausencia de responsabilidades personales y sociales, los déficits en la solución de problemas, así como la falta de sentimientos de culpa y de amor. Asimismo, parecen ser factores de riesgo la juventud y niveles socioeconómicos y culturales bajos. Esto correlaciona, según Torrubia (2004), con la insensibilidad afectiva, considerada por muchos como el núcleo central del trastorno. Sánchez (1995) entiende el trastorno antisocial como un problema relacionado con una mala socialización $\mathrm{y}$, por ello, caracterizado por actitudes antagónicas y falta de autodisciplina. Poseen además una alta extraversión, bajo neuroticismo, baja amabilidad y baja responsabilidad.

Wallace, Vitale y Newman (1999), centrándose en el concepto de "modulación de la respuesta", señalan los fallos de atención que se producen en estos individuos y que son fundamentales para organizar e interpretar la conducta orientada a un fin; y lo que con el tiempo se convierte en un proceso automático que requiere autocomprobación, autoevaluación y autorefuerzo, no se produce; es decir, el problema no radica en los contenidos de los esquemas, pues conocen las reglas, sino en el mal procesamiento de la información dirigida a la consecución de metas.

Desde el modelo de Millon (Millon y col., 2001), la personalidad antisocial se conoce como el patrón activo-independiente y se caracteriza, fundamentalmente, por ser una personalidad agresiva, con distintos grados a lo largo de ese continuo sistemático, abarcando así desde formas leves hasta formas extremadamente graves (Ávila-Espada y Herrero, 1995).

Se trata de un trastorno crónico que con frecuencia se asocia al trastorno de personalidad paranoide, siendo típica la triada antisocial-sádico-pasivo/agresivo (ÁvilaEspada y col., 1995), y al consumo de drogas pues, como afirman Miller, Lynam, Widiger y Leukefeld (2001), recientes estudios han hallado un alto porcentaje de consumidores, abusadores y dependientes de alcohol y otras drogas entre delincuentes psicopáticos. La presencia de trastornos mentales o psicopatológicos y psicopatía incrementa el riesgo de violencia y agresión (Hill, Newman y Rogers, 2004). Se

Revista Española de Investigación Criminológica

Artículo 1, Número 7 (2009) $\quad$ www.criminología.net

ISSN: $1696-9219$ 
caracteriza además por ingresos frecuentes en centros penitenciarios. En su etiología pueden hallarse tanto factores biológicos como de socialización, incluyendo el aprendizaje y las experiencias vitales, tales como la hostilidad parental, graves déficit en los modelos parentales y el reforzamiento de la conducta agresiva y vengativa, potenciados fundamentalmente por la disminución del control de los impulsos (ÁvilaEspada y col., 1995). Tales variables interactúan entre sí dificultando enormemente la posibilidad de definir el peso relativo de unos y otros en el desarrollo del trastorno. Se asocia, además, este trastorno con el neuroticismo, la ausencia de cordialidad y de minuciosidad o escrupulosidad.

\section{Distinción entre psicopatía, sociopatía y trastorno antisocial de la personalidad}

La psicopatía ha recibido distintos nombres a lo largo de la historia, siendo el primer autor en identificarlo Phillipe Pinel (1801-1862) como "manía sin delirio" para referirse a personas cuyas respuestas emocionales eran inusuales y presentaban furias impulsivas, permaneciendo intacta su capacidad de razonamiento. Otros nombres han sido "insania moral”, "egopatía", "sociopatía” y "psicopatía” (Barlow y Durand, 2003). Las psicopatías o trastornos de personalidad antisocial han sido el cajón de sastre de la psicopatología, pues se trata de un trastorno que no permite una fácil asimilación al esquema de síntomas y síndromes. Se trata más bien de problemas referentes a la forma de vida del sujeto (Rodríguez, 1995).

El cambio del término psicopatía por el de trastorno antisocial de la personalidad implica una conceptualización más social del mismo; así, cuando se hace hincapié en la conducta como daño social sería más adecuado utilizar el término trastorno antisocial o sociopatía, mientras que el término psicopatía define mejor las características psicopatológicas de la persona (Luengo y Carrillo, 1995). Para Mora (2004) los sociópatas se diferencian de los psicópatas en algunas características psicológicas y conductuales, a pesar de que ambos manifiesten una clara inadaptación social y agresividad, pero esta última, en el caso de la sociopatía, es reactiva a una situación mientras la agresión y violencia sin motivo aparente, dirigida a conseguir un objetivo concreto, caracteriza a la psicopatía. La existencia de una psicopatía primaria, caracterizada por un temperamento innato, y una psicopatía secundaria, con la

Revista Española de Investigación Criminológica

Artículo 1, Número 7 (2009) $\quad$ www.criminología.net

ISSN: $1696-9219$ 
posibilidad de tener una mayor capacidad de sentir ansiedad y culpa, y que podría ser el resultado de un proceso inadecuado de socialización -aunque sin descartar la influencia de alteraciones biológicas e incluso retrasos madurativos-, incide en la diferenciación entre psicópata y sociópata (Garrido, 2005).

Según De Corral (1996), la preferente denominación de sociopatía obedece a dos motivos: en primer lugar no se trata de alteraciones de índole congénita, y en segundo lugar constituyen una personalidad que, por sus características, conducen a una inadaptación social. Del mismo modo, considera el término psicopatía inadecuado, porque se trata de la denominación de una especie (psicopatías frente a neurosis o psicosis) mediante un nombre genérico, y además hace referencia a aspectos constitucionales, incluso hereditarios, de carácter biológico y psicológico. Por ello, el término psicopatía se sustituye en el DSM-IV por el de trastorno antisocial de la personalidad, constituyéndose la asocialidad en el rasgo central del trastorno, lo que permite diferenciar a los verdaderos psicópatas del resto de delincuentes, es decir, de aquéllos que poseen un correcto funcionamiento dentro de su grupo y son capaces de manifestar sentimientos de culpa, afecto y lealtad. Hare (2003), no obstante, considera que el uso de los términos psicópata o sociópata está determinado por la diferente concepción del trastorno según los distintos profesionales: los que hacen hincapié en que los factores sociales o experiencias infantiles se encuentran en el origen del trastorno utilizan el término sociópata, mientras que aquéllos que entienden que también contribuyen elementos biológicos, psicológicos y genéticos, usan el término psicópata. Sin embargo, para el autor ambos términos definen al mismo individuo, considerándose la psicopatía no como un trastorno mental, sino como un trastorno de la personalidad cuyas características negativas se manifiestan en sus relaciones con los demás, en su afectividad y en su conducta (Raine y Sanmartín, 2000; Cañas, 2003).

No obstante, es un tema controvertido, desde el momento que unos autores señalan como aspecto importante la alteración de la personalidad, mientras que otros señalan la existencia de conductas desviadas. A esta confusión contribuye el que en la literatura científica se mezclen conceptos como criminalidad, sociopatía o trastorno disocial de la personalidad (Ávila-Espada y col., 1995). La dualidad presente en este trastorno tiene importantes repercusiones en su categorización clínica, así como en la

Revista Española de Investigación Criminológica

Artículo 1, Número 7 (2009) $\quad$ www.criminología.net

ISSN: $1696-9219$ 
investigación de su etiología y, por supuesto, en su tratamiento. Tanto es así que los dos grandes sistemas clasificatorios en psicopatología (DSM y CIE) inciden en un aspecto distinto de dicha dualidad: el DSM se centra en el componente comportamental mientras que la CIE lo hace en el aspecto personalista. Hay que tener también en cuenta las diferencias culturales asociadas a la evaluación del trastorno, que son mayores respecto a los síntomas interpersonales y comportamentales, mientras que son más pequeñas respecto a los síntomas afectivos (Cooke, Hart y Michie, 2004).

Así, los criterios que el DSM-IV-TR (APA, 2002) utiliza para la personalidad antisocial básicamente se refieren a conductas observables (por la facilidad que implica para llegar a un acuerdo de diagnóstico entre los clínicos), sobre todo si se comparan con los criterios propuestos por Hare y Cleckley, que se centran sobre todo en rasgos de personalidad subyacentes.

Como puede apreciarse, algunas de las características que propone la APA (2002) no son útiles para discriminar a los psicópatas, puesto que también pueden hallarse en individuos delincuentes no psicópatas. De este modo, y siguiendo a Cantero (1993), quien ya realizaba esta afirmación con respecto al DSM-III-R (APA, 1987), podría ser más adecuado emplear otros instrumentos diagnósticos que se mostraran más precisos para diferenciar a los sujetos que presentan TAP de aquéllos que únicamente exhiben comportamientos antisociales o delictivos. En este sentido, es necesario aclarar que ni todo delincuente es psicópata, ni todo psicópata es delincuente. No obstante, también debe reconocerse que en la última edición del DSM (APA, 2002) empiezan a incluirse algunas de las características propuestas por Cleckley y Hare; es decir, los criterios DSM comienzan a presentar una descripción más en términos de personalidad que de conductas observables, si bien, Farrington (2000) sigue considerando que esta clasificación presenta criterios centrados más en conductas antisociales que en rasgos de personalidad, cuando, en realidad, debería ser al contrario. Así pues, la psicopatía y el trastorno antisocial de la personalidad no son la misma cosa; aunque la relación entre una y otra es incierta, los dos síndromes no se solapan clara y perfectamente (Hare, 1983; Miller y col., 2001). Sin embargo, el mismo Hare (2003) afirma que la psicopatía es un trastorno de la personalidad definido por una serie de conductas y rasgos de la personalidad característicos que socialmente no se consideran positivos. La diferencia

Revista Española de Investigación Criminológica

Artículo 1, Número 7 (2009) $\quad$ www.criminología.net

ISSN: $1696-9219$ 
estriba en que la psicopatía es definida por un conjunto de rasgos de personalidad y conductas socialmente desviadas, mientras que el trastorno de la personalidad antisocial se refiere fundamentalmente a un grupo de conductas delictivas y antisociales, con lo que muchos delincuentes no psicópatas podrían incluirse. La relación entre psicopatía y trastorno antisocial de la personalidad es, por lo tanto, asimétrica, es decir, prácticamente el noventa por ciento de los delincuentes psicópatas cumplen los criterios del trastorno antisocial de la personalidad, pero sólo el veinticinco por ciento de éstos podría diagnosticarse de psicopatía según la Psychopathy Checklist-Revised (PCL-R) 


\section{REFERENCIAS}

American Psychiatric Association (2002). DSM-IV-TR. Manual Diagnóstico y Estadístico de los trastornos Mentales. Texto Revisado. Barcelona: Masson.

Ávila-Espada, A. y Herrero Sánchez, J.R. (1995). La personalidad y sus trastornos: aproximación a la obra de Theodore Millon. Clínica y Salud, 6 (2), 131-159.

Barlow, D. H. y Durand, V. M. (2003). Psicopatologia (3ª ed.). Madrid: Thomson.

Belloch, A. y Fernández-Álvarez, H. (2002). Trastornos de la Personalidad. Madrid: Síntesis.

Blair, R.J.R. (2003). Neurobiological basis of psychopathy. The British Journal of Psychiatry, 182 (1), 5-7.

Bolt, D.M., Hare, R.D., Vitale, J.E. y Newman, J.P. (2004). A multigroup item response theory anlysis of the psychopathy Checklist-Revised. Psychological Assessment, $16(2), 155-168$.

Bueno, A. (1990). La teoría de la criminalidad de H.J. Eysenck: dos lecturas en función de la clase social. Delincuencia, 2 (1), 37-50.

Cantero, F. (1993). )Quién es el psicópata? En V. Garrido, Psicópata (pp. 11-48). Valencia: Tirant lo Blanch.

Cañas, G. (2003). Los expertos creen que si España vigilara a los psicópatas se evitarían muchas muertes. http://galeon.hispavista.com/psicopatas/h7-1.htm

Chico, E. y Ferrando, P.J. (1995). A psychometric evaluation of the revised P scale delinquent and non-delinquent spanish sample. Personality and Individual Differences, 18 (3), 331-337.

Cleckley, H.M. (1941). The Mask of Sanity: an Attempt to Reinterpret the So-Called Psychopathic Personality. St. Louis, Missouri: C.V. Mosby.

Cooke, D.J., Hart, S.D. y Michie, C. (2004). Cross-national differences in the assessment of psychopathy: do they reflect variations in raters' perceptions of symptoms? Psychological Assessment, 16 (3), 335-339.

Corral, P. de (1996). Trastorno antisocial de la personalidad. En E. Echeburúa, Personalidades Violentas (pp. 57-66). Madrid: Ediciones Pirámide.

Eysenck, H.J. (1981). El modelo de condicionamiento en el proceso de socialización. Análisis y Modificación de Conducta, 7 (14-15), 5-29.

Revista Española de Investigación Criminológica

Artículo 1, Número 7 (2009) $\quad$ www.criminología.net

ISSN: $1696-9219$ 
Eysenck, H.J. (1995). Un modelo de personalidad: rasgos generales. En M.D. Avia y M.L. Sánchez, Personalidad: aspectos cognitivos y sociales, (pp. 229-267). Madrid: Pirámide.

Eysenck, H.J. y Eysenck, S.B.G. (1976). Psychoticism as a dimension of personality. London: Hodder and Stoughton.

Farrington, D.P. (2000). Psychosocial predictors of adult antisocial personality and adult convictions. Behavioral Sciences and the Law, 18, 605-622.

Garrido, V. (2004). Tratamiento del agresor psicopático. En J. Sanmartín Espulges (Coord.), El Laberinto de la Violencia: Causas, Tipos y Efectos (pp. 321-330). Barcelona: Ariel.

Garrido, V. (2005). Qué es la Psicología Criminológica. Madrid: Editorial Biblioteca Nueva.

Hare, R.D. (1983). Diagnosis of antisocial personality disorder in two prison populations. American Journal of Psychiatry, 140, 887-890.

Hare, R.D. (1984). La psicopatía: teoría e investigación. Barcelona: Herder.

Hare, R.D. (1991). The Hare Psychopathy Checklist-Revised (PCL-R). Toronto: MultiHealth Systems.

Hare, R.D. (2000). La naturaleza del psicópata: algunas observaciones para entender la violencia depredadora humana. En A. Raine y J. Sanmartín Espulges, Violencia y Psicopatía (pp. 15-58). Barcelona: Editorial Ariel.

Hare, R.D. (2003). Sin Conciencia: El Inquietante Mundo de los Psicópatas que nos Rodean. Barcelona: Ediciones Paidos.

Hicks, B.M., Markon, K.E., Patrick, C.J., Krueger, R.F. y Newman, J.P. (2004). Identifying psychopathy subtypes on the basis of personality structure. Psychological Assessment, 16 (3), 276-288.

Hill, C.D., Newmann, C.S. y Rogers, R. (2004). Confirmatory factor analysis of the Psychopathy Checklist: screening version in offenders with axis I disorders. Psychological Assessment, 16 (1), 90-95.

Karpman, B. (1961). The structure of neurosis: with special differentials between neurosis, psychosis, homosexuality, alcoholism, psychopathy, and criminality. Archives of Criminal Psychodynamic, 4, 599-646.

León Fuentes, J.L. (1996). Psicopatía. En J.L. León Fuentes, Apuntes de Psicopatología de Adultos (pp. 501-534). Sevilla: Kronos. 
Luengo, M.A. y Carrillo, M.T. (1995). La psicopatía. En A. Belloch Fuster, B. Sandín Ferrero y F. Ramos Campos (Eds.), Manual de Psicopatología (vol. 2, pp. 615650). Madrid: McGraw-Hill.

Marcus, D.K., John, S.L. y Edens, J.F. (2004). A taxometric análisis of psychopathic personality. Journal of Abnormal Psychology, 113 (4), 626-635.

Miller, J.D., Lynam, D.R., Widiger, T.A. y Leukefeld, C. (2001). Personality disorders as extreme variants of common personality dimensions: can the five-factors model adequately represent psychopathy? Journal of Personality, 69 (2), 253276.

Millon, T. y Davis, R. (2001). Trastornos de la personalidad en la vida moderna. Barcelona: Masson.

Mora, F. (2004). ¿Cómo Funciona el Cerebro? Madrid: Alianza Ensayo.

Patrick, C.J. (2000). Emociones y psicopatía. En A. Raine y J. Sanmartín, Violencia y psicopatía (pp. 89-118). Barcelona: Ariel.

Pérez, J. (1986). Teoría de Eysenck sobre la criminalidad: el resultado de la investigación. Psiquis, 7, 35-51.

Raine, A. y Sanmartín, J. (2000). Violencia y Psicopatía. Barcelona: Editorial Ariel.

Rodríguez, C. (1995). Clasificación y diagnóstico de la personalidad. Clínica y Salud, 6 (2), 161-173.

Sánchez, M.L. (1995). Los trastornos de personalidad y el modelo de los cinco factores: relaciones empíricas. Clínica y Salud, 6 (2), 175-188.

Stalenhein, E.G. (2001). Personality traits and psychopathy in a forensic psychiatry population. European Journal of Psychiatry, 12 (2), 83-91.

Torrubia, R. (1987). La psicopatía. En J. Pérez (Coord.), Bases psicológicas de la delincuencia y de la conducta antisocial, (pp. 147-164). Barcelona: PPU.

Torrubia, R. (2004). El delincuente. En J. Sanmartín Espulges (Coord.), El Laberinto de la Violencia (pp. 267-281). Barcelona: Ariel.

Wallace, J. F., Vitale, J.E. y Newman, J.P. (1999). Response modulation deficits: implications for the diagnosis and treatment of psychopathy. Journal of Cognitive Psychotherapy, 13, 55-70. 


\section{Curriculum vitae de las autoras}

María José López Miguel es Doctora en Psicología y Experta Universitaria en Criminología por la Universidad de Sevilla. En la actualidad trabaja como docente e investigadora en la Facultad de Psicología de Sevilla, concretamente en el Departamento de Personalidad, Evaluación y Tratamiento Psicológicos, y en el Instituto Andaluz Interuniversitario de Criminología (Sección Sevilla). Sus principales áreas de interés son la delincuencia de tipo violento, la asociación entre conducta adictiva y delictiva y la etiología del comportamiento delictivo en general, fundamentalmente la relacionada con aspectos psicológicos.

María del carmen Núñez Gaitán es Doctora en Psicología y Experta Universitaria en Criminología por la Universidad de Sevilla. En la actualidad trabaja como docente e investigadora en la Facultad de Psicología de Sevilla, concretamente en el Departamento de Personalidad, Evaluación y Tratamiento Psicológicos, y en el Instituto Andaluz Interuniversitario de Criminología (Sección Sevilla). Sus principales áreas de interés son la delincuente en menores, la delincuencia de tipo violento y las consecuencias psicológicas del internamiento en prisión.

\section{CORRESPONDENCIA:}

María José López Miguel. Departamento de Personalidad, Evaluación y Tratamiento Psicológicos. C/ Camilo José Cela, s/n. 41018, Sevilla. Teléfono: 954554359. Fax: 954557807. Correo electrónico: mjmiguel@us.es 